\title{
Modeling Systems with Selectable Structures of Interests and Ideas of the Environment
}

\author{
Gennady P. Vinogradov \\ Tver State Technical University, \\ A. Nikitin Emb., 22 \\ Tver, Russia \\ E-mail:wgp272ng@mail
}

\begin{abstract}
The paper shows that regularities of abandoning an "ideal" rational choice by the subject to transfer to a subjectively rational one are connected with peculiarities of identifying and understanding external conditions and properties of interests. The subject makes choice based on perceptions of a choice situation. Evaluation of satisfaction with it leads to changes in subject's interest structure, and he can choose it. When making decisions, the subject uses three sets of alternatives: control, structural, and identification. The rules for choosing alternatives are formed by a compromise, the problem of choice modeling acquires game content.
\end{abstract}

Keywords: Reflexive governance, decision-making model, decision making, compromise.

\section{Introduction}

The majority of modern information systems are not designed for independent decision-making. Typically, all possible options of such systems behavior are designed by a human and included in them at the design stage. When such system gets into conditions that were not taken into account by its developers, it can lead to emergency termination or severe consequences [5]. One of the approaches to solving this problem is agentoriented programming. An agent is considered as a system that is capable of responding to changes in the external environment, which was not provided by builtin behavioral mechanisms. This property makes the agent's concept an attractive tool for solving many problems that arise in the field of information technologies. Nowadays, researchers are focused on solving the problem of modeling subjectively rational decision making by a natural or artificial entity that has the ability to form goals endogenously based on their interests. The papers [1-5] give a definition of intellectual and purposeful behavior. The papers $[4,11]$ justify that the prerequisites for such behavior are natural and artificial entities (hereinafter an agent) have processes that allow creating internal images of themselves, an influencing environment and determinants of their subjective understanding of choice situations. Such processes are based on input information received from sensors from the environment and experience (knowledge base). Usually, these processes include the stages of perception, observation, understanding and awareness of environment properties and themselves, as well as memories of past situations, representations of desired states. Therefore, the result of fundamental research should be a creation of biotechnical complexes and systems that would act in such a way that their results of work would be impossible to distinguish from actions of a reasonable person.

\section{Follow-up on the Issue}

Traditionally, such studies are classified as the problems of the theory of multi-agent systems (MAS). Its concept was proposed in the mid-1980s. MAC was considered as one of the breakthrough technologies for design and implementation of future distributed intelligent systems. It was proposed to solve problems in the MAC technology in the same way as in the community of living beings, i.e. through the interaction of agents based on self-organization principles. This allowed abandoning traditional programming in the problems of developing complex programs. The agents solved them themselves through interaction using dialogues and data exchange protocols. They were not formed by their developer for subsequent integration. Such paradigm was called the paradigm of interaction-based computing. Its development included significant finances and scientific forces of leading universities and 
IT companies. As a result, the scientists have developed the theoretical foundations for MAS design, a number of methodologies for MAS design, and a number of software tools to support MAS development. They initiated and practically solved the task of scientific substantiation of standards in the field of agents and MAS. Naturally, this approach has become attractive for the tasks of behavioral robotics, both collective and individual. The scientists gave the opportunity to model collective behavior of artificial (robots) and natural entities when performing various missions. This immediately attracted the attention of business and military officers in solving tasks of managing network structure objects. However, despite such tempting opportunities, since 2000 financing of theoretical research and development of tools for MAS creation technological support has been gradually decreasing. So, now it is practically reduced to zero. In the foreground, there are such technologies such as serviceoriented computing, GRID computing, offline computing, cloud computing, foggy computing, etc. They have a different conceptual basis. Although these technologies appeared much later, they were able to quickly push MAC technologies. Therefore, the pace of appearing new inventions of MAC applications has begun to slow down gradually. This fact reflects a real drop in the interest of industrial companies in MAC technology.

The main reason for this fact is related to the proposed solutions for constructing a formal agent model, a software and communication environment architecture, which helps agents interact, as well as implementing agent's communication language. These decisions are practically rejected by those who are interested in practical implementation of MAC technologies. In the theory of agents and MAS, the BDI-model (Belief-Desire-Intention) is used as a basic formal model of an intelligent agent. This model describe knowledge, beliefs, intentions and mechanisms of reasoning in terms of predicate calculus, expanded by modal and temporal operators. An agent based on this architecture was considered as an intellectual entity with its own developed knowledge base, goal-setting means and mechanisms for planning purposeful behavior in an unpredictable external environment. For this purpose, a formal BDI model included various intellectual capacities related to autonomous behavior up to the ability to determine intentions of other agents. The implementation of the BDI-model conceptual framework consisted in active promotion of a logical model enriched by modal and temporal operators. An agent model has become extremely complex. By now, there is a developed logical theory of an agent BDI model that theoretically allows building agents of a high intelligence level. Theoretically, they are able to plan purposeful behavior and autonomous decision-making, to distribute coordination of behavior in complex situations. However, when trying to use theoretical developments in practice for relatively simple applications, there are serious problems, mainly the problems of computational complexity. Even for simple MAC-applications, agent knowledge models are extremely cumbersome, and inference engines that use output in logical calculus with modal and temporal operators are completely unrealizable. Practice has shown that in an acceptable time the interaction of agents might be rather effective only at the level of simple dialogues. In addition, it is also impossible to build models of other agents and the external environment model for making complex decisions.

It should be noted that BDI architecture is a basis of MAC technologies design standard. This standard uses the Agent Communication Language (ACL) to describe the content of agents' messages. This interpretive-type language is powerful and expressive enough; it manipulates ontology concepts and is able to represent the content of messages exchanged by agents in a language close to a natural one. But its logical model generated the same problems of computational complexity, as well as the problem of large communication channel load. In addition, it turned out to be difficult to understand and use for the developers of specific applications. Naturally, research has been started to develop other agent models.

However, the implementation of these properties will be possible after appearing models of decisionmaking by a subject, who has internal images of himself and the party affecting him, and which take into account his subjective understanding of a choice situation [8]. It should be noted that in the normative decision-making theory it is considered that a decision-making process is an uncontrolled factor. Therefore, the development of the theory of multiagent systems is now aimed at solving a set of problems that concentrate around a subjective choice phenomenon [5, 11, 13]. The formal choice theory [12] has been developing through 
abstracting from subjective factors, which led to the creation of a normative decision-making theory by an "ideal" subject. The logic of the choice problem development made it necessary to study how and why the subject "deviates" from normative rationality in the real world $[6,12,15]$. The solution of this problem is connected with the results obtained in the reflexive games theory and the theory of information control of systems with will and intelligence [9, 10, 14]. However, despite many papers in this area [7], the problem remains important.

The development of the idea of subjectively rational choice [3, 8] made it possible to: 1) explain decision making by a subject in specific situations; 2) predict possible reactions of another subject in different situations by a decision maker; 3 ) solve the problem of active forecasting, when a managing party creates the necessary image of the future for a managed party.

Subjectively, a rational choice implies that choice motivation is determined by both external and internal factors. Internal factors reflect subject interests, induced by his needs and the ethical system that he adheres. As shown in [3], estimates of satisfaction with the current purposeful state situation by the subject can lead to changing subject's interests structure and he can choose it. Since subject's preferences in the selection process reflect his interests, it is possible to define a set of $G$ alternative variants of a preference structure, which are called structural alternatives according to [1].

A human brain works with images that he thinks are not accidental, i.e. information perception is directed by a goal. The brain transforms objects into mutually agreed systems, where they establish various types of connections. If this system of connections when used in decision making will obtain planned results, then the brain believes that knowledge: a) is useful; b) is objective; c) is necessary; d) can be trusted.

\section{Control Tasks Solved by an Artificial Autonomous Entity}

In order for an artificial autonomous entity to be considered as the one possessing intelligence, it must demonstrate the ability to create and implement behavioral algorithms that allow it to survive and/or achieve better existence conditions compared to others when it enters an unknown environment. For this purpose, it must have process models in the given environment, which becomes possible if it has an algorithm for obtaining new knowledge, or it can create it. In any case, the stage of identification (model construction) of a choice situation should be before the decision-making process. When making a decision, the entity must be able to distribute available resources between three stages: cognition, solution development, solution implementation. The main resource in this case is time.

It should be assumed a priori that all natural entities have a built-in universal algorithm for finding new knowledge. Such algorithm determines the effectiveness of a artificial entity control system. This fact allows making the following assumptions: 1) an artificial entity should have a built-in universal cognition algorithm; 2) an artificial entity should have a control element; 3) it should have a mechanism for initiating active behavior; 4) it should have a mechanism for optimal management of knowledge storage, representation and transformation; 5) an artificial entity uses accumulated knowledge to fight for survival and create better conditions for its existence; 6) it should have a mechanism for assessing knowledge usefulness and reliability.

The entity must materialize the most useful and reliable knowledge into additional devices that enhance its capabilities, or in built-in reactions (limbic system). Such knowledge transformation allows releasing limited resources of a control element to search for new knowledge.

\section{Initial Assumptions}

1. The subject makes his choice based on ideas about a purposeful state situation.

2. The presentation components reflect different aspects of subject's understanding of a purposeful state situation and form an information structure of representations. We denote the set of possible representation variants by $X$.

3. For a set of environmental conditions $S$, a set of observable environmental conditions satisfy the condition $S \cap X \neq \varnothing$, i.e. subject's representations can contain both an objective and phantom component. 4. The subject chooses structural alternatives depending on estimates of satisfaction by the values of purposeful state situation properties. 
5. Representations are formed based on procedures of perception, awareness and analysis in accordance with subject's cognitive capabilities.

According to the introduced assumptions, the subject uses three sets of alternatives in decision making: control $C$ (operation modes), structural $G$ and identification $X$. Consequently, it can be assumed that there are three virtual parties that choose appropriate alternatives. The rules of choosing such alternatives depending on subject's understanding of the situation and the structure of his interests will be called strategies.

Let decision making has several cyclic steps, and operation modes are chosen at each stage $n=1,2, \ldots$ from the set $C$, depending only on a representation of an environment state $x \in X$. This is due to the fact that the joint supraconscious (intuitive) and a conscious (formal) analysis of an environment state make it possible in multiple iterations to make a decision, vaguely realized at first, and then more clearly formulated and justified one. Moreover, there are restrictions $C_{x} \subseteq C$ on the admissibility of alternatives depending on representations about the environment state $x \in X$. The dynamics of processes in subject's environment is unreachable for direct perception. So, representations about it are formed by using identification procedures, their key point is to choose a representation option depending on the observed state. Moreover, there are some restrictions $X_{s} \subseteq X$ on representation admissibility as identification alternatives depending on the observed states $s \in S$.

Based on these assumptions, following [1], we introduce definitions of strategies. A single-valued transformation $\lambda: X \rightarrow C$ such that $\lambda(x) \in C_{x}, x \in X$, is called a choice or control function; an ordered set $\left(\lambda_{1}, \ldots, \lambda_{n}\right)$ $\equiv \lambda_{1}^{n}$ is a choice strategy on a length horizon $n<\propto$; $\lim \left\{\lambda_{1}^{n}\right\}=\lambda_{1}^{n} \propto$ is a strategy aimed at achieving a local ideal that determines the reason for subject's existence.

A single-valued monotone mapping $\xi: S \rightarrow X$ such that $\xi(s) \in X_{s}, s \in S$, is called an identification function; an ordered set $\left(\xi_{1}, \ldots, \xi_{n}\right) \equiv \xi_{1}^{n}$ is an identification strategy on the length horizon $n<\propto$; the sequence $\left\{\xi_{1}^{n}, n=\right.$ $1,2, \ldots\}$ is an identification strategy on a limited horizon. Since the subject tends to form useful representations, then there is $\lim \left\{\xi_{1}^{n}\right\}=\xi^{\propto}$ for $n \rightarrow \propto$. Since the sets $S$ and $X$ satisfy the condition $|S|>|X|$, then single-valued mapping $\xi: S \rightarrow X$ generates partitioning the set $S$ into subsets.

$$
\xi^{-1}(x)=\bigcup\{s \in S: \xi(s)=x\} \subset S, x \in X .
$$

The subsets $\xi^{-1}(x) \subset S, x \in X$, are connected sets, i.e. any element $s \in \xi^{-1}(x)$ uniquely determines the corresponding representation $x \in X$. Therefore, it is true that the subsets $\xi^{-1}(x) \subset S, x \in X$, make classes of equivalent representations. This will allow using methods of the theory of fuzzy sets to formalize subject's representations, for example, as described in [2].

The structural alternative $\gamma_{n} \in G$ chosen at time $n$ is a structural choice at the $n$-th decision making step; an ordered set $\left(\gamma_{n}, \ldots, \gamma_{1}\right) \equiv \gamma_{1}^{n}$ is a strategy of structural choice on the decision making horizon of the length $n<\propto$; the sequence $\left\{\gamma_{1}^{n}, n=1,2, \ldots\right\}$ is a structural choice strategy on a limited horizon. Since the subject seeks to match his structure of interests to the requirements of an ethical system he has adopted, then there exists $\lim \left\{\gamma_{1}^{n}\right\}=\gamma^{\propto}$ for $n \rightarrow \infty$.

\section{Results}

In contrast to the known models of agent behavior in the project, it is suggested to use a human leader behavioral model (hereinafter the agent) in a particular area as a prototype. For this purpose, the basic approach is observing his behavior in a purposeful state (PS) situation. It is shown that the individuality of the agent is shown in the following assessments: 1) the value of the obtained results that affect his emotional evaluation; 2) the degree of regularity (knowledge) of using operation modes; 3) the degree of influence of environmental factors on his condition; 4) the cost effectiveness of obtaining the desired results. To obtain such assessments, there are the developed appropriate linguistic scales. The problem of constructing a model of subjectively rational choice is solved. It assumes that the person uses three sets of alternatives when making a decision: representations about the environment, operation modes and preferences. The latter is associated with a system of values and norms that are specific to a particular individual. The necessity to include ideas about the state of the environment in the list of alternatives is due to the fact that decisionmaking problems are solved in conditions of uncertainty and weak structuredness. Typically, they are characterized by a big number of vague restrictions, initial data incompleteness, a big number of goals that 
decompose into subgoals, etc. Therefore, a person uses a subjective choice situation model with additional intuitive and associative assumptions. So, the project required to develop a system of concepts for compatibility of a theological approach and an approach based on cause-effect relationships. With regard to the behavior theory (and to the behavior science in general), this approach assumes that the subject domain of the decision theory includes psychological aspects associated with perception, awareness, understanding of choice situation properties and building estimated choice results, communication, conflict, etc. Based on this approach, the obtained results are the following.

1. There is a developed conceptual model of choosing a subject with a subjectively rational form of behavior is provided that the structure of his preferences and notions is not fixed and the subject can choose them. It is a development of the theory of situational management proposed by D.A. Pospelov. Its novelty and development is in the fact that the subject chooses operation modes depending on his attitude to his own state and the state of the choice situation. In both cases, he considers the degree of implementation of his interests, which are qualitative characteristics. When modeling the evolution of subject's behavior, it was the basis for dividing a decision-making process into stages: a) forming ideas about the properties of the choice situation; b) decision-making on an operation mode; c) a technology for implementing a solution and estimated results. It is proposed to evaluate the quality of a decision by the criterion of the specific value of the purposeful state situation based on a result that takes into account the value and probability of the desired states. Purposeful state situations are inaccessible for direct observation and require identification. In the subject's consciousness, they exist in the form of subjective representations. It is proposed to evaluate the quality of representations about the choice situation by the criterion of loss in value. The quality of the technology for solution implementation is evaluated by the criterion of the specific value of a purposeful state situation according to efficiency, taking into account the subjective assessment of subject's costs for solution implementation. It is shown that according to this statement, the subject uses three sets of alternatives when making a decision: a set of strategies for identifying a choice situation when forming representations, a number of operation modes, and a number of options for implementing a solution. The choice of the alternative from the corresponding set is made according to the individual quality criterion. It is proposed for modeling to connect each quality criterion with a virtual operating party that has freedom in choosing within its competence. Subject's interests are dominant for selected parties. For them he is a management center that uses a set of "structural alternatives" reflecting subject's preferences at the time of making a decision. It is suggested to use the assessment of "satisfaction with the choice situation". as a criterion of the agent as an entity. It is shown that in this case, decision making should be considered as a corporate game with non-opposite interests, its "best" solution is to build a sustainable compromise called "equilibrium" on the set of agreed compromise options. The proposed approach allows modeling how a purposeful state situation turns into an aspiration situation for an intelligent agent, which makes it possible to predict its behavior. The novelty of this approach is the ability to assess the effectiveness of reflexive management of subject's behavior prior to its implementation realistically and corresponds to the world level.

2. The author has developed and investigated a system of axioms that define behavior of subjects possessing the properties of activity, autonomy, the ability to form goals and purposeful behavior. The novelty is in determining the development direction of the approach to human behavior as to a system of purposeful (theological) events, and creating systems that have the ability to form the goals of their behavior (evolution) endogenously as a certain sequence of stages when achieving their goals. At the same time, it is a basis for the introduction of a number of functional concepts and grounds for developing the behavioral theory. It makes them objective, measurable and suitable for experiments. It is new that functional characteristics are not input variables. They are objectively derived from what is observed. There is an opportunity to smoothly include in the decision-making process a human intuition, which plays a more important role even in trivial solutions than rational limitations of modern decision-making procedures allow. The limitations imposed by a general logical and mathematical abstraction on decision-making procedures practically exclude the possibility of studying truly creative solutions, which include an 
element of individual choice unlike a logical conclusion. To describe this process, the project provides a developed method for modifying rational behavior models by including reflexive variables that are characteristics of human behavior used in psychology, sociology, etc. Such approach is new, fundamentally different from the known ones and conforms to international standards.

3. The author studies the decision-making model in the case when the subject has full knowledge of the choice situation, is focused on realization of his interests with a subjectively understood "best result", has an idea of estimated results and operation modes by the center and environment, as well as the choice of many tactical structural alternatives. The novelty of this approach is to obtain the conditions for the existence of a set of dynamic equilibria that determine subject's internal goal endogenously with different options of corporate management. 4. The author has developed and investigated the model of the mechanism of building coordinated decisions in systems with an endogenously formed goal and with the adopted system of interests and internal values. Reflexive processes of making intuitive decisions within the framework of postnonclassical rationality assume that a person uses the position of an "observer", "researcher" or "implementer" in relation to his actions and thoughts. This allows the team to use the ability of each person to be in the researcher position in relation to another "character", his actions and thoughts when making coherent intuitive decisions. Such approach to modeling reflexive decision-making processes is new and has not previously been considered in the theory of MAS systems. The novelty of the approach is connected with creation of a theoretical basis for constructing third generation recommended systems. These systems develop solutions based on subjective models of user's decision making, take into account the assessments of his interests, preferences, motivation and obligations, as well as the semantics of the context that accompanies decision making as user's subjective representations. This approach conforms to international standards in constructing intelligent information technologies for decision support that are primarily aimed at their personification.

\section{A Decision-making Model with a Changing Preference Structure}

According to [3], the criterion for choosing a management strategy means the expected specific value of a purposeful state from the result. Its formalization has the form of the utility function $E \varphi^{g}(C \times S \times X)$, which depends on the structural alternative $g \in G$ as on a parameter. Since the control process starts with a certain situation $x \in X$, then the criterion $E_{\varphi_{n}}\left(\lambda_{1}^{n} \mid \gamma_{1}^{n}\right)$ will also depend on the situation $x \in X$ as on the initial condition. Since in this case the set of situations $X$ is finite, then the criterion $E \varphi n\left(\lambda_{1}^{n} \mid \gamma_{1}^{n}\right)$ will be finally represented by a vector in the space $R^{X}$ of dimension $|\mathrm{X}|$. Its components are written in the form $E \varphi n\left(\lambda_{1}^{n} \mid \gamma_{1}^{n}\right)(x), x \in X$. The subject experiences an emotional experience caused by the choice result. Therefore, the quality of the structural choice strategy $\gamma_{1}^{n}$ should be described as a criterion with the meaning of "satisfaction with choice". Therefore, it is natural to describe the quality of a strategy $\gamma_{1}^{n}$ by convolution of the expected utility vector $\operatorname{E\varphi n}\left(\lambda_{n} \mid \gamma_{1}^{n}\right) \in R^{X}$ into some functional $\mu: R^{X} \rightarrow R^{1}$. Then the criterion of the strategy quality $\gamma_{1}^{n}$ can be written in the form:

$$
\mu_{n}\left(\lambda_{1}^{n} \mid \gamma_{1}^{n}\right)=\mu\left(E \varphi_{n}\left(\lambda_{1}^{n} \mid \gamma_{1}^{n}\right)\right) \in R^{1}
$$

The subject associates the quality of his representations with the assessments of the possibility of achieving the desired states from controlling $c \in C$, as well as the possibility of expanding the set $C \uparrow$ by including effective alternatives in it. The paper [4] uses the terms of the linguistic variable "utility", which are built on the values $E \varphi_{n}\left(\lambda_{1}^{n} \mid \gamma_{1}^{n}\right)$, as a criterion for estimating representations. At the same time, utility estimates will depend on control strategies $\lambda_{1}^{n}$, structural choice $\gamma_{1}^{n}$ as from the given conditions. Let us denote the criterion "utility" as $\psi_{n}\left(\xi_{1}^{n} \mid \lambda_{1}^{n}, \gamma_{1}^{n}\right)$. Since the identification process starts with a certain state $s \in S$, then this criterion will depend on the state $s \in S$ that is given as the initial condition. Since in this case the set of states $S$ is finite, the identification criterion will be represented by a vector $\psi n\left(\xi_{1}^{n} \mid \lambda_{1}^{n}, \gamma_{1}^{n}\right)$ in $R^{S}$ space of dimension $|S|$.

The introduced criteria in the purposeful state situation involves identification of appropriate information structures or models that allow making an appropriate choice.

We assume the existence of an information structure of representations $I$ that reflects subject's knowledge 
and experience about: operation (management) modes, own interests and preferences, the dynamics of transition of the environment into different states. Then we can assume that there is a structural transformation of this structure into an information structure that provides the possibility of constructing a specific value criterion $E \varphi_{n}\left(\lambda_{1}^{n} \mid \gamma_{1}^{n}\right)$ and a domain model. Such transformation will be called a "specific value transformation", the induced information structure will be called an "informational structure of the specific value of a purposeful state situation from the result" and denoted as $U=U(I)$. The technique of such transformation is described in [3].

Similarly, if there is a structural transformation of the structure $I$ into an information structure that provides the possibility of constructing an identification criterion $\psi n\left(\xi_{1}^{n} \mid \lambda_{1}^{n}, \gamma_{1}^{n}\right)$ and a model of identification procedures, then such transformation will be called an "identification transformation" and denoted by $R$. The induced information structure will be called an "identification information structure" and denoted as $R=R(I)$

Subject's representations about the purposeful state situation are subjective and qualitative, constructed based on observations and analysis of the process of environment transition under control of $c \in C$ into various states $s \in S$. Let us denote the rule for such transition a $q^{g}(S \mid S \times C)$ from $S \times C$ into $S$. In fact, to evaluate possible outcomes, the subject uses the constructed from the results of the identification strategy $\xi_{1}^{n}$ model $Q^{g}(X \mid X \times C)$ from $X \times C$ into $X$. When building, he takes into account management strategies $\lambda_{1}^{n}$, structural choice strategies $\gamma_{1}^{n}$, or he defines such strategies. It means that the transformation of the actual function $q^{g}(S \mid S \times C)$ into a function of understanding by the subject of surrounded processes $Q^{g}(X \mid X \times Y)$ is possible only in a posteriori mode, depending on the used strategies $\left(\lambda_{1}^{n}, \gamma_{1}^{n}, \xi_{1}^{n}\right)$. Such transformation and construction of the expected specific value criterion $E \varphi_{n}\left(\lambda \mid \gamma_{1}^{n}\right)$ is possible when "utility" information structures are formed successively depending on the strategies used. This condition will be written as $U_{n}=U\left(\lambda_{1}^{n}, \gamma_{1}^{n}, \xi_{1}^{n}\right)(I), n=1,2, \ldots$ Since this condition is necessary for the formation of the expected utility criterion and a domain model, it must be indicated whenever it is used. It should be noted that the criterion $E \varphi_{n}\left(\lambda_{1}^{n} \mid \gamma_{1}^{n}\right)$ implicitly depends on the identification strategy $\xi_{1}^{n}$ by introducing the induced structure $U_{n}$ into the choice model. As noted above, the criterion $\mu_{n}\left(\gamma_{1}^{n} \mid \lambda_{1}^{n}\right) \in R^{1}$ for structural choice quality is determined by the convolution of the criterion $\operatorname{E\varphi n}\left(\lambda_{1}^{n} \mid \tau_{1}^{n}\right) \in R^{X}$. The generality of the information structure of their formation allows us to write down:

$$
\begin{gathered}
E \varphi n\left(\lambda_{1}^{n} \mid \xi_{1}^{n}\right), \mu_{n}\left(E \varphi n\left(\xi_{1}^{n} \mid \lambda_{1}^{n}\right)\right), \\
U_{n}=U\left(\lambda_{1}^{n}, \gamma_{1}^{n}, \xi_{1}^{n}\right)(I) .
\end{gathered}
$$

The construction of an identification criterion requires using some function that would have the meaning of "utility". For this purpose it is necessary to construct verbal estimates on the values of the utility function $E \varphi^{g}(S \times X \times Y)$. The required transformation exists and can be performed in a priori mode (i.e. before choosing solutions). Such transformation is determined by the subject with respect to a fuzzy measure that can be constructed if a function $q^{g}(S \mid S \times C)$ from $S \times C$ in $S$ is given. Since its analogue in subject's consciousness has the form $Q^{g}(X \mid X \times C)$ and he can define it uniquely in the information structure $I$, then no additional transformations are required. The construction of the "representation utility" function exhausts a necessary structural transformation. Such transformation will be called the structural transformation of "identification" and denoted by $R$. The induced information structure will be called the information structure of the "representations utility" and denoted by $R=R(I)$.

Taking these considerations into account, we finally write the identification criterion:

$$
\left\{\psi_{n}\left(\xi_{1}^{n} \mid \lambda_{1}^{n}, \gamma_{1}^{n}\right), \quad R=R(I) .\right.
$$

Based on the introduced definitions and constructions it is possible to say that quality criteria for strategies are different and interdependent. So, the problem of choice has a game content and is reduced to finding a stable compromise between the desire to maximize the expected specific value of a purposeful state by the result and to minimize possible losses from misconceptions under conditions of limited resource which is time. Such compromise is called equilibrium.

Since the information structure of the "specific value" $U n=U\left(\lambda_{1}^{n}, \gamma_{1}^{n}, \xi_{1}^{n}\right)(I)$, under which the criterion $\mu_{n}\left(E \varphi_{n}\left(\gamma_{1}^{n} \mid \lambda_{1}^{n}\right)\right)$ is built, should be formed sequentially depending on the strategies used, then the desired equilibria will be interdependent not only at 
each stage $n=1,2, \ldots$ of solution formation, but they will also depend on the decisions chosen in the preceding steps. Considering this fact, equilibria are $\begin{array}{lll}o & o & o\end{array}$ dynamic. A triple of strategies $\left\{\lambda_{1}^{n}, \gamma_{1}^{n}, \xi_{1}^{n}\right\}$ satisfying the following conditions:

$$
\begin{aligned}
& E \varphi_{n}\left(\begin{array}{c}
0 \\
\lambda_{1}^{n} \mid
\end{array} \mid \begin{array}{l}
\gamma_{1}^{n} \\
)
\end{array} \geq E \varphi_{n}\left(\lambda_{1}^{n} \mid \begin{array}{l}
0 \\
\gamma_{1}^{n}
\end{array}\right),\right. \\
& \forall \lambda_{1}^{n}, \mu_{n}\left(\lambda_{1}^{n}{ }_{1}^{n} \begin{array}{l}
0 \\
\gamma_{1}^{n}
\end{array}\right) \geq \mu_{n}\left(\lambda_{1}^{n} \mid \begin{array}{l}
\gamma_{1}^{n} \\
n
\end{array}\right) \text {, } \\
& \forall \gamma_{1}^{n}, U_{n}\left(\lambda_{1}^{n}, \gamma_{1}^{n}, \xi_{1}^{n}\right)(I),
\end{aligned}
$$

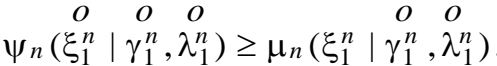

$$
\begin{aligned}
& R=R(I), n=1,2, \ldots . .
\end{aligned}
$$

is called dynamic equilibria.

According to the assumptions, the number of decisionmaking cycles is unlimited. Then dynamic equilibria should make sense, including for $n \rightarrow \infty$. For this purpose, it is natural to set the following additional conditions:

1) when $n \rightarrow \infty$, the criteria for the quality of strategies should tend to some limits;

2) such limits should not depend on the initial conditions.

Since the criteria are not specified explicitly, the fulfillment of conditions requires setting the desired properties and specifying criteria of an explicit type that satisfy these properties. According to the assumptions, the criteria of the quality of stationary strategies $\lambda^{n}, \gamma^{n}, \xi^{n}$ for $n \rightarrow \infty$ have limits, then the triple of $o$
stationary strategies $\left(\lambda^{\infty}, \gamma^{\infty}, \xi^{\infty}\right)$ is called stationary equilibria if there are limits for it that satisfy the following conditions:

$$
\begin{aligned}
& \varphi\left(\lambda^{\infty} \mid \gamma^{\infty}\right) \geq \varphi n\left(\lambda^{\infty} \mid \gamma^{\infty}\right), \forall \lambda^{\infty}, \\
& \mu \begin{array}{c}
o \\
\mu\left(\gamma^{\infty} \mid \lambda^{\infty}\right) \geq \mu_{n}\left(\gamma^{\infty} \mid \lambda^{\infty}\right), \forall \gamma^{\infty},
\end{array} \\
& \left.U=\begin{array}{c}
o \\
U\left(\lambda^{\infty},\right.
\end{array}, \gamma^{\infty}, \xi^{\infty}\right)(I), \\
& \left.\begin{array}{c}
o \\
\psi\left(\xi^{\infty} \mid \lambda^{\infty}, \gamma^{\infty}\right.
\end{array}\right) \geq \psi\left(\xi^{\infty} \mid \begin{array}{cc}
o & o \\
\lambda^{\infty} & \gamma^{\infty}, \forall \xi^{\infty},
\end{array}\right.
\end{aligned}
$$

Thus, the problem of choice modeling is finding a compromise between the desire to achieve the maximum expected specific value by the result and the minimum loss from misconceptions, taking into account their mutual dependence. According to the principle of equilibrium solutions, it must be "not improved" simultaneously for all interest components. When reaching such compromise, it can be affirmed that subject's interests are realized with the "best result". In this case if the dynamic equilibria satisfy the requirements of asymptotic stationarity, then it can be also affirmed that subject's interests are realized with the "best result" including for $n \rightarrow \infty$. Therefore, dynamic equilibria determine the meaning and method of implementing of interests with the "best result". Taking into account this fact, dynamic equilibria naturally determine the internal goal in decision making.

\section{The Algorithm for Extracting New Knowledge}

1) To create variants of initial ideas about the situation of choice, in which there is at least one reliable link of the required knowledge with varying degree of utility using available methods of passive and active identification. To save this information in a knowledge base. To use a priori information in similar choice situations. 2) To analyze information in the knowledge base and make a reasonable assumption about the direction for increasing the utility of representations of a choice situation (formation of knowledge hypothetical links). This supposed direction will be not exact, but fuzzy (probabilistic, expressed in a linguistic form). It will have the form of a range of possible directions of search. 3) To search for new knowledge in selected areas, starting with the most probable ones. To assess the degree of their utility and the probability of achieving the desired states. If the variant of a possible purposeful state situation is considered satisfactory, then a new useful link of knowledge is found. Move to the next step. Otherwise, return to step 2). 4) To save information about a new link in a knowledge base and move to step 2).

The abovementioned makes it relevant to describe information flow types that occur in agent's mind from his environment and how to measure them. These information flows are aimed at forming agent's representations of the situation of choice. Naturally, the formation of representations is connected with energy and time consumption. So, there must be something that makes the agent do it. Psychologists call it instincts; we will call it the built-in mechanisms of unconditional execution. They are: 
- the desire of any entity (natural or artificial) to maintain its identity (the survival axiom);

- the mechanism of launching active behavior and maintaining its intensity (the emotional stimulation axiom);

- the ability to create models of choice situations, to form modes of behavior, to remember and use them for survival (the cognition axiom).

The author proposes a decision-making model for an agent capable of forming an internal goal and using subjective ideas about the situation of choice. It is shown that the purpose of choice is to maximize the specific value of a choice situation by the result. The result of choice is determined by agent's ideas about the situation of choice and his own interests. When making decisions, he uses three sets of alternatives: control $C$ (operation modes), structural $G$ and identification $X$. Therefore, we can assume the existence of three virtual parties that select the appropriate alternatives that are equilibrium strategies.

\section{Acknowledgements}

The work has been financially supported by RFBR, project no. 170100728 .

\section{References}

1. V. V. Baranov, Dynamic equilibriums in the problems of stochastic management and decision-making under uncertainties. J. of Computer and Systems Sciences Int. 3 (2002) 77-93 (in Russ.).

2. P. A. Borisov, G. P. Vinogradov and N. A. Semenov, Integration of neural network algorithms, nonlinear dynamic models and methods of fuzzy logic in prediction tasks. J. of Computer and Systems Sciences Int. 1 (2008) 78-84 (in Russ.).

3. G. P. Vinogradov and V. N. Kuznetsov, Modeling agent's behavior based on subjective perceptions on the situation of choice. Iskusstvenny intellekt $i$ prinyatie resheny (Artificial Intelligence and Decision Making), 3 (2011) 58-72 (in Russ.).

4. G. P. Vinogradov, G. P. Shmatov and D. A. Borzov, Formation of agent's representations on a domain in the situation of choice. Software and Systems. 2(110) (2015) 83-94 (in Russ.).

5. V. I. Gorodetsky, V. V. Samoylov and D. V. Trotsky, Basic ontology of collective behavior of Autonomous agents and its expansion. J. of Computer and Systems Sciences Int. 5 (2015) 102-121 (in Russ.).

6. D. Kaneman, P. Slovik and A. Tverski, Decision Making under Uncertainty: Rules and Prejudices (Kharkov, Gumanitarny tsentr, 2005) (in Russ.).

7. A. V. Karpov, General Subjective Choice Psychology: Structure, Process, Genesis (Institut psikhologii RAN, Yaroslavl State Univ., Yaroslavl, 2000) (in Russ.)

8. V. A. Lefevr, Conflicting Structures (Sovetskoe radio, Moscow, 1973) (in Russ.).

9. D. A. Novikov and A. G. Chkhartishvili, Reflexive Games (Sinteg, Moscow, 2003) (in Russ.).

10. D. A. Novikov and A. G. Chkhartishvili, Applied models of information management (IPU RAN, Moscow, 2004) (in Russ.)

11. M. Polanyi, Personal Knowledge. Towards a PostCritical Philosophy (Univ. of Chicago Press, 1974).

12. H. A. Simon, The Sciences of the Artificial (MIT Press, 1970).

13. R. Solso, Cognitive Psychology (Allyn and Bacon, Boston, 2001).

14. A. G. Chkhartishvili, Game-theoretic Models of Information Management (PMSOFT, Moscow, 2004) (in Russ.).

15. W. Edwards and A. Tversky, Decision Making (Harmoudsworth Middlesex, England Penguin Books, 1972). 